UWThPh-1998-19

DFTT $15 / 98$

hep-ph/9804421

January 23,2020

\title{
Four-Neutrino Mixing and Big-Bang Nucleosynthesis
}

\author{
S.M. Bilenky \\ Joint Institute for Nuclear Research, Dubna, Russia, and \\ INFN, Sezione di Torino, and Dipartimento di Fisica Teorica, Università di Torino, \\ Via P. Giuria 1, I-10125 Torino, Italy \\ C. Giunti \\ INFN, Sezione di Torino, and Dipartimento di Fisica Teorica, Università di Torino, \\ Via P. Giuria 1, I-10125 Torino, Italy \\ W. Grimus and T. Schwetz \\ Institute for Theoretical Physics, University of Vienna, \\ Boltzmanngasse 5, A-1090 Vienna, Austria
}

\begin{abstract}
We investigate the Big-Bang Nucleosynthesis constraints on neutrino mixing in the framework of the two four-neutrino schemes that are favored by the results of neutrino oscillation experiments. We discuss the implications of these constraints for terrestrial short and long-baseline neutrino oscillation experiments and we present some possibilities of testing them in these experiments. In particular, we show that from the Big-Bang Nucleosynthesis constraints it follows that the $\stackrel{(-)}{\nu}) \rightarrow \stackrel{(-)}{\nu}$ 位 transition is severely suppressed in short-baseline experiments, whereas its oscillation amplitude in long-baseline experiments is of order 1 . We also propose a new parameterization of the four-neutrino mixing matrix $U$ which is appropriate for the schemes under consideration.
\end{abstract}

14.60.Pq, 14.60.St, 26.35.+c

Typeset using REVTEX 


\section{INTRODUCTION}

The problem of the masses and mixing of neutrinos (see Refs. [1]) is the first priority problem of neutrino physics. Many experiments searching for neutrino oscillations and neutrinoless double-beta decay and investigating the high-energy part of the tritium beta-spectrum are going on or are under preparation. At present, indications that neutrinos are massive and mixed have been found in solar neutrino experiments (Homestake [5], Kamiokande [6], GALLEX [7], SAGE [8] and Super-Kamiokande [9,10]), in atmospheric neutrino experiments (Kamiokande [11], IMB [12], Soudan [13] and Super-Kamiokande [14, 10]) and in the LSND experiment [15]. From the analyses of the data of these experiments in terms of neutrino oscillations it follows that there are three different scales of neutrino mass-squared differences:

$$
\begin{gathered}
\Delta m_{\mathrm{sun}}^{2} \sim 10^{-5} \mathrm{eV}^{2}(\mathrm{MSW}) \text { or } \Delta m_{\mathrm{sun}}^{2} \sim 10^{-10} \mathrm{eV}^{2} \text { (vac. osc.) [16,17, } \\
\Delta m_{\mathrm{atm}}^{2} \sim 5 \times 10^{-3} \mathrm{eV}^{2} \\
\Delta m_{\mathrm{SBL}}^{2} \sim 1 \mathrm{eV}^{2},
\end{gathered}
$$

where $\Delta m_{\mathrm{SBL}}^{2}$ is the neutrino mass-squared difference relevant for short-baseline (SBL) experiments, whose allowed range is determined by the positive results of the LSND experiment. The two possibilities for $\Delta m_{\text {sun }}^{2}$ correspond, respectively, to the MSW [19] and to the vacuum oscillation solutions of the solar neutrino problem.

Thus, at least four light neutrinos with definite masses (with only three active flavor neutrinos, $\left.\nu_{e}, \nu_{\mu}, \nu_{\tau}\right)$ must exist in nature in order to accommodate the results of all neutrino oscillation experiments. This means that there exists at least one non-interacting sterile neutrino [20 26]. In this case, a left-handed neutrino field $\nu_{\alpha L}(\alpha=e, \mu, \tau, s)$ is a mixture of the left-handed components $\nu_{k L}$ of the four fields of neutrinos with definite masses $m_{k}$ $(k=1, \ldots, 4)$ :

$$
\nu_{\alpha L}=\sum_{k=1}^{4} U_{\alpha k} \nu_{k L} \quad(\alpha=e, \mu, \tau, s),
$$

where $U$ is the $4 \times 4$ unitary mixing matrix.

In Ref. 222] we considered the schemes with four massive neutrinos and we have shown that from six possible mass spectra of four massive neutrinos only the following two mass spectra with two pairs of close masses separated by a gap of about $1 \mathrm{eV}$ (the "LSND gap") are compatible with all existing data (see also Ref. [23]):

$$
\underbrace{\overbrace{m_{1}<m_{2}}^{\text {atm }} \ll \overbrace{m_{3}<m_{4}}^{\text {sun }}}_{\text {SBL }} \quad \text { and }
$$

$$
\underbrace{\overbrace{m_{1}<m_{2}}^{\text {sun }} \ll \overbrace{m_{3}<m_{4}}^{\text {atm }}}_{\text {SBL }} .
$$

In scheme A, $\Delta m_{\text {atm }}^{2} \equiv m_{2}^{2}-m_{1}^{2}$ and $\Delta m_{\text {sun }}^{2} \equiv m_{4}^{2}-m_{3}^{2}$ are the mass-squared differences relevant for atmospheric and solar neutrino oscillations, respectively, whereas in scheme B we have $\Delta m_{\text {sun }}^{2} \equiv m_{2}^{2}-m_{1}^{2}$ and $\Delta m_{\text {atm }}^{2} \equiv m_{4}^{2}-m_{3}^{2}$. In both schemes $\Delta m_{\mathrm{SBL}}^{2} \equiv m_{4}^{2}-m_{1}^{2}$ is relevant for neutrino oscillations in short-baseline experiments. 
In this paper we study the compatibility of these two schemes with an upper bound $N_{\nu} \lesssim 3.9$ (see Ref. 27]) on the effective number $N_{\nu}$ of light neutrinos relevant in Big-Bang Nucleosynthesis (BBN) (see, for example, Ref. [28]). We derive the constraints on some elements of the neutrino mixing matrix $U$ that follow from $N_{\nu} \lesssim 3.9$ (see also Ref. [23]) and we discuss some possibilities to check these constraints in future short-baseline (SBL) and long-baseline (LBL) neutrino oscillation experiments.

Let us notice that there was recently some discussion in the literature about the validity of the upper bound $N_{\nu} \lesssim 3.9$ (see Ref. [27]). This discussion was initiated by conflicting results of different measurements of the deuterium abundance in high-redshift hydrogen clouds [29, 30]. The situation is not completely clarified, but there seems to exist now a strong case in favor of $N_{\nu} \lesssim 3.9$ [27].

Let us define the quantities

$$
c_{\alpha} \equiv \sum_{k=1,2}\left|U_{\alpha k}\right|^{2} \quad(\alpha=e, \mu, \tau, s)
$$

Okada and Yasuda [23] have shown that the bound $N_{\nu} \lesssim 3.9$ implies stringent limits on $c_{s}$ in scheme A and $1-c_{s}$ in scheme $\mathrm{B}$. This means that the solar neutrino deficit is explained by small angle MSW $\nu_{e} \rightarrow \nu_{s}$ transitions 2 and the atmospheric neutrino anomaly by $\nu_{\mu} \rightarrow \nu_{\tau}$ transitions (see Ref. [18]). In this paper we have reanalyzed the constraints on the parameter $c_{s}$ that follow from $\mathrm{BBN}$. We have obtained more stringent upper bounds on $c_{s}$ in scheme $\mathrm{A}$ and $1-c_{s}$ in scheme $\mathrm{B}$ than those presented in Ref. 23] and we have investigated the effects of these constraints for SBL and LBL neutrino oscillations, some of which can be tested in the near future.

The plan of the paper is as follows. In Section II we summarize the formalism of SBL neutrino oscillations in the schemes A and B (for more details see Refs. [21,22]). In Section III] we investigate in detail the dependence of $N_{\nu}$ on $c_{s}$. From the BBN upper bound on $N_{\nu}$ we obtain upper bounds on $c_{s}$ in scheme A and $1-c_{s}$ in scheme B. In Sections [V] and $\mathrm{V}$ we obtain various general relations between SBL and LBL oscillation amplitudes and $c_{s}$. These relations can be useful to check the BBN constraints and to test the hypothesis of existence of a sterile neutrino. In Section $\nabla$ we also introduce a new parameterization of the mixing matrix that is suitable for the schemes under consideration.

\footnotetext{
${ }^{1}$ For $N_{\nu} \geq 4$ the schemes $\mathrm{A}$ and $\mathrm{B}$ are trivially compatible with BBN.

${ }^{2}$ The vacuum oscillation and the large angle MSW solutions of the solar neutrino problem are disfavored by the data in the case of $\nu_{e} \rightarrow \nu_{s}$ transitions (see Refs. [31, 32, 16]).

${ }^{3}$ This explanation of the atmospheric neutrino anomaly is consistent with the recent results of the first long-baseline reactor experiment CHOOZ [33] in which no indications in favor of $\bar{\nu}_{e}$ disappearance were found.
} 


\section{NEUTRINO OSCILLATIONS IN SBL EXPERIMENTS}

In the schemes under consideration the probability of $\nu_{\alpha} \rightarrow \nu_{\beta}$ transitions $(\beta \neq \alpha)$ and the survival probability of $\nu_{\alpha}$ in SBL experiments are given by [21]

$$
\begin{aligned}
& P_{\nu_{\alpha} \rightarrow \nu_{\beta}}=\frac{1}{2} A_{\alpha ; \beta}\left(1-\cos \frac{\Delta m_{\mathrm{SBL}}^{2} L}{2 p}\right), \\
& P_{\nu_{\alpha} \rightarrow \nu_{\alpha}}=1-\frac{1}{2} B_{\alpha ; \alpha}\left(1-\cos \frac{\Delta m_{\mathrm{SBL}}^{2} L}{2 p}\right),
\end{aligned}
$$

with the oscillation amplitudes

$$
\begin{aligned}
& A_{\alpha ; \beta}=4\left|\sum_{i} U_{\beta i} U_{\alpha i}^{*}\right|^{2}, \\
& B_{\alpha ; \alpha}=4\left(\sum_{i}\left|U_{\alpha i}\right|^{2}\right)\left(1-\sum_{i}\left|U_{\alpha i}\right|^{2}\right),
\end{aligned}
$$

where the index $i$ runs over the values 1,2 or 3,4 .

In the following, we will consider values of $\Delta m_{\mathrm{SBL}}^{2}$ in the wide range

$$
10^{-1} \mathrm{eV}^{2} \leq \Delta m_{\mathrm{SBL}}^{2} \leq 10^{3} \mathrm{eV}^{2} .
$$

The direct comparison of the regions in the $A_{\mu ; e}-\Delta m_{\mathrm{SBL}}^{2}$ plane allowed at $90 \%$ CL by the results of the LSND experiments with the regions excluded at $90 \%$ CL by the results of other SBL experiments indicate a considerably smaller allowed range for $\Delta m_{\mathrm{SBL}}^{2}$ :

$$
0.27 \mathrm{eV}^{2} \lesssim \Delta m_{\mathrm{SBL}}^{2} \lesssim 2.2 \mathrm{eV}^{2}
$$

The results of the combined analysis of the data of all SBL experiments presented in Ref. [34] show that the lower bound $\Delta m_{\mathrm{SBL}}^{2} \gtrsim 0.27 \mathrm{eV}^{2}$ is robust (if the LSND indication is correct), whereas values of $\Delta m_{\mathrm{SBL}}^{2}$ larger than $2.2 \mathrm{eV}^{2}$ maybe are not excluded.

Taking into account the results of solar and atmospheric neutrino experiments, from the exclusion curves of SBL disappearance experiments at each possible value of $\Delta m_{\mathrm{SBL}}^{2}$ we have 22

$$
\begin{aligned}
& c_{e} \leq a_{e}^{0} \quad \text { and } \quad 1-c_{\mu} \leq a_{\mu}^{0} \quad \text { in scheme A, } \\
& 1-c_{e} \leq a_{e}^{0} \quad \text { and } \quad c_{\mu} \leq a_{\mu}^{0} \quad \text { in scheme B , }
\end{aligned}
$$

where

$$
a_{\alpha}^{0} \equiv \frac{1}{2}\left(1-\sqrt{1-B_{\alpha ; \alpha}^{0}}\right) \quad(\alpha=e, \mu)
$$

and $B_{\alpha ; \alpha}^{0}$ is the upper bound for the amplitude $B_{\alpha ; \alpha}$ that can be obtained from the exclusion plots of SBL reactor and accelerator disappearance experiments. From the 90\% CL exclusion curves of the Bugey [35] $\bar{\nu}_{e} \rightarrow \bar{\nu}_{e}$ reactor experiment and of the CDHS [36] and CCFR 37] $\nu_{\mu} \rightarrow \nu_{\mu}$ accelerator experiments it follows that $a_{e}^{0}$ is small $\left(a_{e}^{0} \lesssim 4 \times 10^{-2}\right)$ for $\Delta m_{\mathrm{SBL}}^{2}$ in the range (2.5) and $a_{\mu}^{0} \lesssim 10^{-1}$ for $\Delta m_{\mathrm{SBL}}^{2} \gtrsim 0.5 \mathrm{eV}^{2}$ (see Fig. 1 of Ref. [38]). 


\section{BBN CONSTRAINTS ON 4-NEUTRINO MIXING}

In this section we derive the $\mathrm{BBN}$ constraints on the elements of the mixing matrix in the two schemes A and B. We follow the standard arguments 39 42,23 leading to the BBN constraints on the elements of the mixing matrix $U$ if a sterile neutrino exists. According to the standard BBN scenario (see, for example, Ref. [28]) a lepton asymmetry can be neglected and the active neutrinos are in chemical equilibrium. Thus, the following considerations are valid for a temperature range $T_{\text {dec }} \lesssim T \lesssim 100 \mathrm{MeV}$ with the decoupling temperature $T_{\mathrm{dec}} \sim 2 \mathrm{MeV}$ for electron neutrinos and $T_{\mathrm{dec}} \sim 4 \mathrm{MeV}$ for muon and tau neutrinos. In this temperature region, the effective potentials of neutrinos due to coherent forward scattering in the primordial plasma are given by $43,41,42$

$$
V_{e} \equiv V=-6.02 G_{F} p \frac{T^{4}}{M_{W}^{2}}, V_{\mu, \tau}=\xi V \quad \text { and } \quad V_{s}=0
$$

where $p$ is the neutrino momentum, which henceforth will be replaced by its temperature average $\langle p\rangle \simeq 3.15 T$, and $\xi=\cos ^{2} \theta_{W} /\left(2+\cos ^{2} \theta_{W}\right) \simeq 0.28$. The propagation of neutrinos is governed by the effective hamiltonian [19]

$$
\begin{aligned}
H_{\nu} & =\frac{1}{2 p} U \operatorname{diag}\left(m_{1}^{2}, m_{2}^{2}, m_{3}^{2}, m_{4}^{2}\right) U^{-1}+\operatorname{diag}(V, \xi V, \xi V, 0) \\
& =U^{\prime} \operatorname{diag}\left(E_{1}, E_{2}, E_{3}, E_{4}\right) U^{\prime-1}
\end{aligned}
$$

where $E_{1}, E_{2}, E_{3}, E_{4}$ are the effective energy eigenvalues of neutrinos in matter and $U^{\prime}$ is the corresponding effective mixing matrix. The amount of sterile neutrinos present at nucleosynthesis can be calculated using the differential equation [39]

$$
\frac{d n_{\nu_{s}}}{d t}=\frac{1}{2} \sum_{\alpha=e, \mu, \tau}\left\langle P_{\nu_{\alpha} \rightarrow \nu_{s}}\right\rangle_{\mathrm{coll}} \Gamma_{\nu_{\alpha}}\left(1-n_{\nu_{s}}\right)
$$

where $n_{\nu_{s}}$ is the number density of the sterile neutrino relative to the number density of an active neutrino in equilibrium and $\Gamma_{\nu_{\alpha}}$ are the collision rates of the active neutrinos, including elastic and inelastic scattering [42], given by

$$
\Gamma_{\nu_{e}}=4.0 G_{F}^{2} T^{5} \quad \text { and } \quad \Gamma_{\nu_{\mu}, \nu_{\tau}}=B \Gamma_{\nu_{e}} \quad \text { with } \quad B=0.722 .
$$

Let us notice some important characteristics of Eq.(3.3):

i. The probabilities for $\nu_{\alpha} \rightarrow \nu_{s}$ transitions are averaged over the collision time $t_{\text {coll }}=$ $1 / \Gamma_{\nu_{e}}$ and also $n_{\nu_{s}}$ has to be considered as such an averaged quantity.

\footnotetext{
${ }^{4}$ In the absence of a lepton asymmetry the neutrinos and antineutrinos evolve identically and hence only neutrinos are considered here.
} 
ii. This equation should approximately hold for non-resonant and adiabatic resonant neutrino transitions, as long as the active neutrinos are in chemical equilibrium and $t_{\text {osc }} \ll t_{\text {coll }} \ll t_{\text {exp }}$ is fulfilled. The characteristic expansion time of the universe $t_{\exp }$ is given by $t_{\exp }=1 / H$ where $H$ is the Hubble parameter, which is related to the temperature $T$ by $H=-\dot{T} / T \simeq 0.69(T / 1 \mathrm{MeV})^{2} \mathrm{~s}^{-1}$.

iii. The condition $t_{\mathrm{osc}} \ll t_{\text {coll }}$ means that neutrino oscillations have to be fast relative to the collision time. The relation $\Gamma_{\nu_{e}} / H \simeq 1.2(T / 1 \mathrm{MeV})^{3}$ shows that for temperatures larger than $2 \mathrm{MeV}$ the collision time is always much smaller than the expansion time [39].

iv. Chemical equilibrium for the active neutrinos is maintained at temperatures larger than the neutrino decoupling temperature even in the presence of sterile neutrinos, provided the relevant mixing angles in the primordial plasma are not too large [42].

Using the relation $H=-\dot{T} / T$, the evolution equation (3.3) for $n_{\nu_{s}}$ can be rewritten as

$$
\frac{d n_{\nu_{s}}}{d T}=-\frac{1}{2 H T} \sum_{\alpha=e, \mu, \tau}\left\langle P_{\nu_{\alpha} \rightarrow \nu_{s}}\right\rangle_{\text {coll }} \Gamma_{\nu_{\alpha}}\left(1-n_{\nu_{s}}\right) .
$$

Since by definition $N_{\nu}$ is the effective number of massless neutrino species at $T_{\mathrm{dec}}$, in order to get a constraint on the mixing of sterile neutrinos we need to calculate the value of $n_{\nu_{s}}$ at $T_{\text {dec }}$ produced by neutrino oscillations. With the initial condition $n_{\nu_{s}}\left(T_{i}\right)=0\left(T_{i} \sim 100\right.$ $\mathrm{MeV}$ ), the integration of Eq.(3.5) gives [44]

$$
n_{\nu_{s}}\left(T_{\mathrm{dec}}\right)=1-\exp \left(-\int_{T_{\mathrm{dec}}}^{T_{i}} \frac{1}{2 H T} \sum_{\alpha=e, \mu, \tau}\left\langle P_{\nu_{\alpha} \rightarrow \nu_{s}}\right\rangle_{\mathrm{coll}} \Gamma_{\nu_{\alpha}} d T\right) \equiv 1-\exp (-F) .
$$

Imposing the upper bound

$$
n_{\nu_{s}}\left(T_{\mathrm{dec}}\right) \leq \delta N \equiv N_{\nu}-3
$$

one obtains the condition

$$
F \leq|\ln (1-\delta N)| .
$$

Since $c_{e}$ is small in scheme A and $1-c_{e}$ is small in scheme B (see Eqs.(2.7) and (2.8)), they do not have any effect on neutrino oscillations during BBN. Hence in this Section we use the approximation $c_{e}=0$ in scheme $\mathrm{A}$ and the approximation $1-c_{e}=0$ in scheme $\mathrm{B}$ [23]. In this case, the elements $U_{e i}$ and $U_{s i}(i=1, \ldots, 4)$ of the mixing matrix can be chosen real and can be parameterized by

$$
\begin{aligned}
& \left(U_{e i}\right)=\left(0,0, c_{\theta}, s_{\theta}\right), \quad\left(U_{s i}\right)=\left(s_{\varphi} s_{\chi},-s_{\varphi} c_{\chi},-c_{\varphi} s_{\theta}, c_{\varphi} c_{\theta}\right) \quad \text { in scheme A, } \\
& \left(U_{e i}\right)=\left(c_{\theta}, s_{\theta}, 0,0\right), \quad\left(U_{s i}\right)=\left(s_{\varphi} s_{\theta},-s_{\varphi} c_{\theta},-c_{\varphi} s_{\chi}, c_{\varphi} c_{\chi}\right) \quad \text { in scheme B, }
\end{aligned}
$$

with the abbreviations $c_{\rho} \equiv \cos \rho$ and $s_{\rho} \equiv \sin \rho$ for $\rho=\theta, \chi, \varphi$ and $0 \leq \varphi \leq \pi / 2$. In both schemes A and B we have $c_{s}=\sin ^{2} \varphi$. 
Then, it can be shown that [23]

$$
\begin{aligned}
\sum_{\alpha=e, \mu, \tau}\left\langle P_{\nu_{\alpha} \rightarrow \nu_{s}}\right\rangle_{\mathrm{coll}} \Gamma_{\nu_{\alpha}}=\frac{B \Gamma_{\nu_{e}}}{2} & \left\{\frac{D^{2} \sin ^{2} 2 \chi}{1-2 D \xi x_{\mathrm{atm}} \cos 2 \chi+D^{2}\left(\xi x_{\mathrm{atm}}\right)^{2}}\right. \\
& \left.+\frac{4 c_{s}\left(1-c_{s}\right)}{1-2 \xi x_{\mathrm{SBL}}\left(1-2 c_{s}\right)+\left(\xi x_{\mathrm{SBL}}\right)^{2}}\right\}
\end{aligned}
$$

where

$$
x_{\sigma} \equiv \frac{2 p V}{\Delta m_{\sigma}^{2}}=-2.18 \times 10^{-7}\left(\frac{\Delta m_{\sigma}^{2}}{1 \mathrm{eV}^{2}}\right)^{-1}\left(\frac{T}{1 \mathrm{MeV}}\right)^{6} \quad(\sigma=\mathrm{atm}, \mathrm{SBL})
$$

and $p$ is replaced by its temperature average. With the definitions

$$
D \equiv c_{s} \text { in scheme } \mathrm{A} \text { and } D \equiv 1-c_{s} \text { in scheme } \mathrm{B},
$$

Eq. (3.11) holds in both schemes. The two terms in Eq. (3.11) correspond to oscillations due to $\Delta m_{\mathrm{atm}}^{2}$ and $\Delta m_{\mathrm{SBL}}^{2}$, respectively. Oscillations due to $\Delta m_{\text {sun }}^{2}$ are neglected in Eq.(3.11) because their contribution to $F$ (see Eq.(3.6)) is at least two orders of magnitude smaller for $T>T_{\mathrm{dec}}$.

Using the expression (3.11) one can find an analytic form of the integral (3.6) and the condition (3.8) gives the bound

$$
920\left(\frac{\Delta m_{\mathrm{SBL}}^{2}}{1 \mathrm{eV}^{2}}\right)^{1 / 2} c_{s} \sqrt{1-c_{s}}+33\left(\frac{\Delta m_{\mathrm{atm}}^{2}}{10^{-2} \mathrm{eV}^{2}}\right)^{1 / 2} \frac{\sin ^{2} 2 \chi}{\sqrt{1+\cos 2 \chi}} D^{3 / 2} \leq|\ln (1-\delta N)| .
$$

With the definitions (3.13) this bound holds for both schemes, provided the conditions laid down in i.-iv. are fulfilled.

Let us now discuss the implications of the bound (3.14). In order to understand if this bound is compatible with a large $D$, we first consider the possibility that $D$ is close to one. In this case we have $\left(U_{\mu 1}, U_{\mu 2}\right) \sim(\cos \chi, \sin \chi)$ in scheme $\mathrm{A}$ and $\left(U_{\mu 3}, U_{\mu 4}\right) \sim(\cos \chi, \sin \chi)$ in scheme B. This means that, in order to accommodate the atmospheric neutrino anomaly, $\sin ^{2} 2 \chi$ cannot be small. However, this is in contradiction with the inequality (3.14) because of the second term in the left-hand side. Therefore, we conclude that the bound (3.14) implies that $D$ is small, i.e. $c_{s}$ is small in scheme A and $1-c_{s}$ is small in scheme B.

Let us now check the validity of this reasoning considering the oscillations due to $\Delta m_{\mathrm{atm}}^{2}$ which generate the second term in Eq.(3.14) and taking into account that the method considered here only holds for non-resonant neutrino oscillations or adiabatic transitions through a resonance. Looking at Eq.(3.11) we see that there is a resonant behaviour of the oscillations due to $\Delta m_{\mathrm{atm}}^{2}$ at the temperature where the relation $D \xi x_{\mathrm{atm}}=\cos 2 \chi$ is satisfied. Since $x_{\text {atm }}$ is negative, this is only possible if $\cos 2 \chi$ is negative. However, since we do not know the sign of $\cos 2 \chi$, we have to check if the resonance is crossed adiabatically in the case of a negative sign. A measure of the adiabaticity of the resonance is given by the parameter

$$
q_{\mathrm{atm}}=\left|\frac{1}{\Delta E_{\mathrm{atm}}} \frac{d \chi^{\prime}}{d t}\right|_{\max }=1.23 \times 10^{-4}\left(\frac{\Delta m_{\mathrm{atm}}^{2}}{10^{-2} \mathrm{eV}^{2}}\right)^{-1 / 2} \frac{\sin 2 \chi}{\sqrt{D}(1+\cos 2 \chi)^{3 / 2}}
$$


where $\Delta E_{\text {atm }}$ and $\chi^{\prime}$ are the oscillation frequency and the effective mixing angle in matter. If $q_{\text {atm }} \ll 1$ the adiabatic crossing of the resonance is guaranteed. Clearly, for $D \sim 1$ and $\sin ^{2} 2 \chi \sim 1$ this condition is fulfilled. Furthermore, oscillations due to $\Delta m_{\text {atm }}^{2}$ occur around the temperature $T_{\text {atm }} \sim 7 D^{-1 / 6} \mathrm{MeV}$, where $D\left|\xi x_{\text {atm }}\right| \sim 1$. One can show that for $D \sim 1$ the relation $t_{\text {osc }} \ll t_{\text {coll }}$ holds for resonant and non-resonant oscillations due to $\Delta m_{\text {atm }}^{2}$ and thus the conditions for the validity of Eq.(3.3) and all relations derived from it are fulfilled as well. Consequently, the exclusion of the case $D \sim 1$ with the help of the second term in Eq.(3.14) is correct. It is interesting to note that no conclusions can be drawn from the second term in Eq.(3.14) if $D \lesssim 0.1$ because then $T_{\text {atm }} \gtrsim 10 \mathrm{MeV}$ and in this temperature region one has $t_{\mathrm{osc}} \gg t_{\text {coll }}$ and therefore no oscillations due to $\Delta m_{\text {atm }}^{2}$ are possible because of strong quantum damping.

Let us now examine the implications of the first term in Eq.(3.14). The presence of this term implies that either $c_{s}$ or $\sqrt{1-c_{s}}$ has to be very small. As we have just shown considering the second term in Eq.(3.14), in scheme $\mathrm{A} c_{s}$ is small. This implies that $1-2 c_{s}>0$ and only non-resonant oscillations are possible (see the last term in Eq.(3.11)). Hence, Eq.(3.14) is valid in scheme $\mathrm{A}$ and the first term gives the bound

$$
c_{s} \leq 1.1 \times 10^{-3}\left(\frac{\Delta m_{\mathrm{SBL}}^{2}}{1 \mathrm{eV}^{2}}\right)^{-1 / 2}|\ln (1-\delta N)| \equiv \mathcal{B}^{(\mathrm{A})} .
$$

On the other hand, considering the second term in Eq.(3.14), we have shown that in scheme B the quantity $1-c_{s}$ is small. Therefore, in scheme $\mathrm{B}$ we have $1-2 c_{s}<0$ and a resonance occurs at the temperature $T_{\text {res }}=16\left(\Delta m_{\mathrm{SBL}}^{2} / 1 \mathrm{eV}^{2}\right)^{1 / 6}\left|1-2 c_{s}\right|^{1 / 6} \mathrm{MeV}$, where $\xi x_{\mathrm{SBL}}=1-2 c_{s}$. One can show that this resonance is not passed adiabatically, hence the conditions for the validity of Eq.(3.3) are not fulfilled and the first term of Eq.(3.14) does not apply. In this case the amount of sterile neutrinos produced at the resonance through non-adiabatic transitions can be calculated with the formula 45,46]

$$
n_{\nu_{s}}=\frac{1}{2}-\left(\frac{1}{2}-P_{L Z}\right) \cos 2 \varphi_{b} \cos 2 \varphi_{a} \simeq 1-P_{L Z}
$$

where $\varphi_{b}\left(\varphi_{a}\right)$ is the effective mixing angle before (after) the resonance. The last approximation in Eq.(3.17) follows from the fact that $\cos 2 \varphi_{b} \simeq 1$ because of the high effective neutrino potential before the resonance and $\cos 2 \varphi_{a} \simeq \cos 2 \varphi=1-2 c_{s} \simeq-1$ for small $1-c_{s}$. The quantity $P_{L Z}=\exp (-Q)$ is the Landau-Zener probability [47 and $Q$ is given by [45,42,46]

$$
\begin{aligned}
Q \equiv \frac{\pi^{2}}{2} \frac{\delta t}{t_{\mathrm{osc}}} & =\frac{2 \pi}{\hbar} \frac{c_{s}\left(1-c_{s}\right)}{\left|1-2 c_{s}\right|}\left|V\left(\frac{d x_{\mathrm{SBL}}}{d t}\right)^{-1}\right|_{T_{\mathrm{res}}} \\
& \simeq 9.2 \times 10^{4} \frac{c_{s}\left(1-c_{s}\right)}{\left|1-2 c_{s}\right|^{3 / 2}}\left(\frac{\Delta m_{\mathrm{SBL}}^{2}}{1 \mathrm{eV}^{2}}\right)^{1 / 2}
\end{aligned}
$$

where $\delta t$ is the half-width of the resonance defined in Ref. [41]. Demanding again that $n_{\nu_{s}} \leq \delta N$ one obtains the condition $Q \leq|\ln (1-\delta N)|$ which gives the bound

$$
1-c_{s} \leq 1.1 \times 10^{-5}\left(\frac{\Delta m_{\mathrm{SBL}}^{2}}{1 \mathrm{eV}^{2}}\right)^{-1 / 2}|\ln (1-\delta N)| \equiv \mathcal{B}^{(\mathrm{B})}
$$


in scheme B. The linear approximation of the potential used to derive the Landau-Zener formula is valid if the potential changes slowly in the resonance region. At the resonance one obtains $|V /(d V / d t)| / \delta t \sim\left|1-2 c_{s}\right| / 2 \sqrt{c_{s}\left(1-c_{s}\right)}$ which is large for small $1-c_{s}$. Hence the linear approximation should apply.

In the following table we show the values of the bounds $\mathcal{B}^{(\mathrm{A})}$ and $\mathcal{B}^{(\mathrm{B})}$ obtained from Eqs.(3.16) and (3.19) and the LSND lower bound $\Delta m_{\mathrm{SBL}}^{2} \gtrsim 0.27 \mathrm{eV}^{2}$ (see Eq.(2.6) for different values of $\delta N$ :

\begin{tabular}{cc|cccccccc} 
& $\delta N$ & 0.9 & 0.8 & 0.7 & 0.6 & 0.5 & 0.4 & 0.3 & 0.2 \\
\hline scheme A: & $\mathcal{B}^{(\mathrm{A})} / 10^{-3}$ & 4.8 & 3.4 & 2.5 & 1.9 & 1.4 & 1.1 & 0.7 & 0.5 \\
scheme B: & $\mathcal{B}^{(\mathrm{B})} / 10^{-5}$ & 4.9 & 3.4 & 2.6 & 1.9 & 1.5 & 1.1 & 0.8 & 0.5
\end{tabular}

The bounds (3.16) for the non-resonant oscillations in scheme A and (3.19) for the resonant case in scheme $\mathrm{B}$ are in rough agreement with the corresponding numerical integrations of the evolution equations of an ensemble of oscillating neutrinos which take into account 2-neutrino oscillations [42,46].

Let us now summarize our findings. The constraints on the parameter $c_{s}$ in scheme A (Eq.(3.16)) and $1-c_{s}$ in scheme B (Eq.(3.19)) has been derived in both schemes from the oscillations due to $\Delta m_{\mathrm{SBL}}^{2}$ at temperatures of the order $T \sim 16 \mathrm{MeV}$ where $\xi\left|x_{\mathrm{SBL}}\right| \sim 1$. The second term in Eq.(3.14), which is generated by the oscillations due to $\Delta m_{\text {atm }}^{2}$, only serves to exclude large $D$. The transitions of active into sterile neutrinos due to $\Delta m_{\mathrm{SBL}}^{2}$ are non-resonant in scheme A, whereas they are resonant in scheme B, leading to a stronger constraint on $1-c_{s}$ than the constraint on $c_{s}$ in scheme A (see table (3.20)). We have obtained stronger bounds than Ref. 23] because we have taken into account the complete collision rates (3.4) presented in Ref. [42], which are nearly an order of magnitude larger than those used in Ref. [23]. Furthermore, our method shows that the dependence of the bound on $c_{s}$ in scheme $\mathrm{A}$ and $1-c_{s}$ in scheme $\mathrm{B}$ on the allowed number of neutrino species $N_{\nu}$ is logarithmic [44.

We want to emphasize that our calculation of the amount of sterile neutrinos brought into equilibrium by oscillations before the onset of BBN is based upon equation (3.3) in scheme A and upon the Landau-Zener approximation in scheme B. Therefore, the numbers given in table (3.20) should be viewed as order of magnitude estimates of the upper bounds on the parameter $c_{s}$ in scheme $\mathrm{A}$ and $1-c_{s}$ in scheme $\mathrm{B}$.

Concluding this section, we would like to make some comments on the non-standard BBN scenario with a non-zero lepton asymmetry presented in Ref. [48]. There it has been shown that for a certain range of the mixing parameters and for resonant transitions due to $\Delta m_{\mathrm{SBL}}^{2}$ a lepton asymmetry as small as $10^{-10}$ can be amplified up to $10^{-2}$ and in this way transitions at a lower temperature (like the transitions due to $\Delta m_{\text {atm }}^{2}$ in the schemes under consideration) can be prevented. Such a scenario would not change our bound for $1-c_{s}$ in scheme $\mathrm{B}$, but could have some effect on our considerations for scheme A. It $c_{s}$ is large in scheme $\mathrm{A}$, a resonance due to $\Delta m_{\mathrm{SBL}}^{2}$ occurs and, if a large lepton asymmetry is generated, the oscillations due to $\Delta m_{\text {atm }}^{2}$ are suppressed and the second term on the left-hand side of Eq.(3.14) is not valid. Hence a large parameter $c_{s}$ cannot be excluded. Therefore, contrary to our discussion in this section, in this case we would have two possibilities for scheme A: either $c_{s}$ is small and has to obey the bound (3.16) or $c_{s}$ is close to one and $1-c_{s}$ has to 
obey the same bound as given in Eq.(3.19) for scheme B. However, judging from the results of Ref. [48] it has yet to be shown that with $c_{s}$ close to one a realistic scenario meeting all experimental constraints can actually be achieved in scheme A and we leave this problem to future investigation.

\section{TERRESTRIAL NEUTRINO OSCILLATION EXPERIMENTS AND STERILE NEUTRINO MIXING}

As we have shown in the previous Section, BBN suggests that the parameter $c_{s}$ in scheme A $\left(1-c_{s}\right.$ in scheme B) is small. In this Section we present some possibilities to obtain information on the parameter $c_{s}$ from future results of SBL and LBL neutrino oscillation experiments. We consider explicitly scheme A, but the same results are valid in scheme B after the replacement of $c_{s}$ with $1-c_{s}$.

Let us start with the consideration of $\nu_{\mu} \rightarrow \nu_{\tau}$ oscillations, that are presently searched for in the CHORUS [49] and NOMAD [50] experiments and will be searched for in the COSMOS [51] experiment. From Eq.(2.3), for the amplitude of $\nu_{\mu} \rightarrow \nu_{\tau}$ oscillations we have the upper bound

$$
A_{\mu ; \tau} \leq 4\left(1-c_{\mu}\right)\left(1-c_{\tau}\right)
$$

The unitarity of the mixing matrix implies that

$$
\sum_{\alpha} c_{\alpha}=2
$$

and Eq.(4.1) can be rewritten as

$$
A_{\mu ; \tau} \leq 4\left(1-c_{\mu}\right)\left[c_{e}+c_{s}-\left(1-c_{\mu}\right)\right]
$$

Since the quantity $1-c_{\mu}$ satisfies the inequality (2.7), if

$$
c_{e}+c_{s} \geq 2 a_{\mu}^{0}
$$

we have

$$
A_{\mu ; \tau} \leq 4 a_{\mu}^{0}\left(c_{e}+c_{s}-a_{\mu}^{0}\right) \leq 4 a_{\mu}^{0}\left(a_{e}^{0}+c_{s}\right) .
$$

On the other hand, if the inequality

$$
c_{e}+c_{s} \leq 2 a_{\mu}^{0}
$$

is satisfied, for the amplitude $A_{\mu ; \tau}$ we have

$$
A_{\mu ; \tau} \leq\left(c_{e}+c_{s}\right)^{2} \leq 4\left(a_{\mu}^{0}\right)^{2} .
$$

As it is seen from Eqs.4.5) and (4.7), if it will be found that the amplitude $A_{\mu ; \tau}$ satisfies the inequality 


$$
A_{\mu ; \tau}>4\left(a_{\mu}^{0}\right)^{2},
$$

it will mean that the parameter $c_{s}$ must satisfy the inequality

$$
c_{s} \geq 2 a_{\mu}^{0}-a_{e}^{0}
$$

and is in general not small. In Fig.1 we have plotted the values of $4\left(a_{\mu}^{0}\right)^{2}$ (solid curve) and $B_{\mu ; \mu}^{0}$ (dashed curve) obtained from the 90\% CL exclusion curves of the CDHS [36] and CCFR [37] experiments in the range (2.5) of $\Delta m_{\mathrm{SBL}}^{2}$, that covers the LSND-allowed region. The dashed curve representing $B_{\mu ; \mu}^{0}$ constitutes an upper bound for $A_{\mu ; \tau}$ due to the conservation of probability. Therefore, if the inequality (4.8) is satisfied, $A_{\mu ; \tau}$ must lie between the solid and dashed curves. In Fig.1 1 we have also plotted the most recent exclusion curve presented by the CHORUS collaboration [52] (dash-dotted curve), the expected final sensitivity of the CHORUS [49] and NOMAD [50] experiments (dash-dot-dotted curve) and the expected sensitivity of the COSMOS [51] experiment (dotted curve). One can see that a substantial part of the region in which the inequality (4.8) is satisfied is already ruled out by the present CHORUS exclusion curve, a large part will be excluded when the final sensitivities of the CHORUS and NOMAD experiments will be reached and almost all the region will be excluded if the COSMOS experiment will not find $\nu_{\mu} \rightarrow \nu_{\tau}$ oscillations.

In Fig.2 we have plotted the values of $2 a_{\mu}^{0}-a_{e}^{0}$ obtained from the 90\% CL exclusion curves of the Bugey [35], CDHS [36] and CCFR [37] experiments. One can see that for the values of $\Delta m_{\mathrm{SBL}}^{2}$ in which $a_{e}^{0}<2 a_{\mu}^{0}$, if the inequality (4.8) is satisfied $c_{s}$ must be large and incompatible with the BBN upper bound. On the other hand, for the values of $\Delta m_{\mathrm{SBL}}^{2}$ in which $a_{e}^{0}>2 a_{\mu}^{0}, c_{s}$ does not need to be large (the inequality (4.4) is satisfied because $c_{e}$ can be larger than $2 a_{\mu}^{0}$ ) and there is no contradiction with the BBN upper bound on $c_{s}$ even if the inequality (4.8) is satisfied. If $A_{\mu ; \tau}$ will be measured and found to satisfy the inequality (4.8), also $\Delta m_{\mathrm{SBL}}^{2}$ will be determined with some accuracy. In this case, depending on the allowed range of $\Delta m_{\mathrm{SBL}}^{2}$, it will be possible to decide if a small $c_{s}$ compatible with the BBN upper bound is excluded or not. Note, however, that for the LSND range (2.6) of $\Delta m_{\mathrm{SBL}}^{2}$ the parameter $c_{s}$ must be large if the inequality (4.8) is satisfied.

Furthermore, if some future experiment will measure a value of $A_{\mu ; \tau}$ such that Eq.(4.8) is satisfied, then the inequality (4.5) will imply in addition the lower bound

$$
c_{s} \geq \frac{A_{\mu ; \tau}}{4 a_{\mu}^{0}}-a_{e}^{0},
$$

which is more stringent than the lower bound (4.9) if $A_{\mu ; \tau}>8\left(a_{\mu}^{0}\right)^{2}$.

Let us now consider the possibility that a future experiment will measure a small value for the amplitude $A_{\mu ; \tau}$, i.e. such that the inequality (4.7) is satisfied. This measurement will not give any information on the value of $c_{s}$. In this case, the BBN bound derived in Section III implies that $c_{s} \simeq 0$ and for the amplitude $A_{\mu ; \tau}$ we have the upper bound

$$
A_{\mu ; \tau} \leq\left(a_{e}^{0}\right)^{2} .
$$

The numerical values of this upper bound in the range (2.5) of $\Delta m_{\mathrm{SBL}}^{2}$ obtained from the 90\% CL exclusion curve of the Bugey [35] experiment is shown in Fig. (3 (solid curve), together with the most recent exclusion curve presented by the CHORUS collaboration [52] 
(dash-dotted curve), the expected final sensitivity of the CHORUS 49] and NOMAD [50] experiments (dash-dot-dotted curve) and the expected sensitivity of the COSMOS [51] experiment (dotted curve).

Thus, we have seen that the investigation of $\nu_{\mu} \rightarrow \nu_{\tau}$ oscillations in the region of $\Delta m_{\mathrm{SBL}}^{2}$ that includes the LSND-allowed region could allow to obtain information on the value of the parameter $c_{s}$ that is important for BBN.

Let us consider now the possibility to obtain information on the parameter $c_{s}$ from the data of inclusive $\nu_{\mu} \rightarrow \nu_{\mu}$ and $\bar{\nu}_{e} \rightarrow \bar{\nu}_{e}$ experiments. From Eqs.(2.4) and (2.7) it follows that

$$
\begin{aligned}
c_{e} & =\frac{1}{2}\left(1-\sqrt{1-B_{e ; e}}\right), \\
1-c_{\mu} & =\frac{1}{2}\left(1-\sqrt{1-B_{\mu ; \mu}}\right) .
\end{aligned}
$$

Furthermore, from Eq.(4.2) we have

$$
c_{s} \geq\left(1-c_{\mu}\right)-c_{e} .
$$

From Eqs.(4.12)-(4.14) we obtain the inequality

$$
c_{s} \geq \frac{1}{2}\left(\sqrt{1-B_{e ; e}}-\sqrt{1-B_{\mu ; \mu}}\right) .
$$

The amplitude $B_{e ; e}$ is small in the whole range (2.5) of $\Delta m_{\mathrm{SBL}}^{2}$. If the parameter $c_{s}$ is small as suggested by BBN, also the amplitude $B_{\mu ; \mu}$ must be small. The existing data do not exclude, however, large values of $B_{\mu ; \mu}$ for $\Delta m_{\mathrm{SBL}}^{2} \lesssim 0.3 \mathrm{eV}^{2}$. Hence, our analysis shows that the investigation of inclusive $\nu_{\mu} \rightarrow \nu_{\mu}$ transitions for $\Delta m_{\mathrm{SBL}}^{2} \lesssim 0.3 \mathrm{eV}^{2}$ would be interesting for the check of the constraint that follows from BBN.

Let us now consider the amplitude $A_{e ; s}$ of $\nu_{e} \rightarrow \nu_{s}$ oscillations. From the upper bound

$$
A_{e ; s} \leq 4 c_{e} c_{s} \leq 4 a_{e}^{0} c_{s},
$$

which follows from Eqs.(1.6), (2.3) and (2.7), it is clear that the BBN bound on $c_{s}$ implies that $A_{e ; s}$ is extremely small. In this case the amplitudes measured in SBL $\nu_{e}$ disappearance experiments and SBL $\nu_{e} \rightarrow \nu_{\mu}$ and $\nu_{e} \rightarrow \nu_{\tau}$ appearance experiments are related by

$$
B_{e ; e} \simeq A_{\mu ; e}+A_{e ; \tau} .
$$

The experimental check of this relation allows to test the BBN bound on $c_{s}$. Indeed, if SBL $\nu_{e}$ disappearance experiments will find neutrino oscillations with a lower limit $B_{e ; e}^{(\min )}$ for the amplitude $B_{e ; e}$ such that $B_{e ; e}^{(\min )}>A_{\mu ; e}^{(\max )}+A_{e ; \tau}^{(\max )}$, where $A_{\mu ; e}^{(\max )}$ and $A_{e ; \tau}^{(\max )}$ are the experimental upper limit for $A_{\mu ; e}$ and $A_{e ; \tau}$, respectively, it will mean that $A_{e ; s}>0$. In this case, taking into account that $B_{e ; e}=A_{\mu ; e}+A_{e ; \tau}+A_{e ; s}$ and that for small $B_{e ; e}$ we have $c_{e} \simeq B_{e ; e} / 4$, the inequality $A_{e ; s} \leq 4 c_{e} c_{s}$ gives the lower bound

$$
c_{s} \geq \frac{A_{e ; s}}{4 c_{e}} \simeq 1-\frac{A_{\mu ; e}+A_{e ; \tau}}{B_{e ; e}} \geq 1-\frac{A_{\mu ; e}^{(\max )}+A_{e ; \tau}^{(\max )}}{B_{e ; e}^{(\min )}} .
$$


If, for example, it will be found that $B_{e ; e}^{(\min )} \simeq 10^{-2}, A_{\mu ; e}^{(\max )} \simeq 5 \times 10^{-3}$ and $A_{e ; \tau}^{(\max )} \ll A_{\mu ; e}^{(\max )}$, Eq.(4.18) will imply the lower bound $c_{s} \gtrsim 0.5$, which is incompatible with the BBN upper bound.

$\mathrm{CP}$ violation in long-baseline neutrino oscillation experiments can also be used to check the BBN constraint on $c_{s}$, though in practice this possibility is more remote. It has been shown in Ref. [25] that the absolute value of the CP-violating parameter $I_{\mu \tau}=4 \operatorname{Im}\left[U_{\mu 1} U_{\tau 1}^{*} U_{\mu 2}^{*} U_{\tau 2}\right]$ (in scheme A, whereas $I_{\mu \tau}=4 \operatorname{Im}\left[U_{\mu 3} U_{\tau 3}^{*} U_{\mu 4}^{*} U_{\tau 4}\right]$ in scheme B) could be as large as $2 / 3 \sqrt{3}$, which is the maximal value allowed by the unitarity of the mixing matrix. With the method discussed in Ref. [25] one can show that

$$
\left|I_{\mu \tau}\right| \leq\left(c_{e}+c_{s}\right) \sqrt{1-c_{e}-c_{s}}
$$

for small $c_{e}+c_{s}$. From the upper bound $c_{e} \leq a_{e}^{0}$ (see Eq.(2.7)) and the BBN upper bound $c_{s} \leq \mathcal{B}^{(\mathrm{A})}$ (see Eq.(3.16)) we have

$$
\left|I_{\mu \tau}\right| \leq\left(a_{e}^{0}+\mathcal{B}^{(\mathrm{A})}\right) \sqrt{1-a_{e}^{0}-\mathcal{B}^{(\mathrm{A})}} .
$$

Consequently, taking into account that $a_{e}^{0}$ is small, the BBN upper bound on $c_{s}$ implies that $\mathrm{CP}$ violation in the $\nu_{\mu} \rightarrow \nu_{\tau}$ channel is suppressed. On the other hand, if CP violation will be observed in LBL $\nu_{\mu} \rightarrow \nu_{\tau}$ experiments, using Eq.(1.19) it will be possible to set a lower bound for $c_{s}$. Analogous results are valid in scheme $\mathrm{B}$ with the replacements $c_{e} \rightarrow 1-c_{e}$, $c_{s} \rightarrow 1-c_{s}$ and $\mathcal{B}^{(\mathrm{A})} \rightarrow \mathcal{B}^{(\mathrm{B})}$.

\section{A PARAMETERIZATION OF THE 4-NEUTRINO MIXING MATRIX}

In this Section we propose a parameterization of the $4 \times 4$ mixing matrix $U$ which is suitable for the two schemes $\mathrm{A}$ and $\mathrm{B}$ that allow to accommodate all existing neutrino oscillation data. In these schemes the neutrinos $\nu_{1}, \nu_{2}$ and the neutrinos $\nu_{3}, \nu_{4}$ give separate contributions to all observables. Thus, it is natural to consider separately the elements $U_{\alpha i}$ with $i=1,2$ and the elements $U_{\alpha k}$ with $k=3$, 4 . In the following we will stick to scheme A for the actual presentation. The formulas in scheme B can be obtained with the exchanges $1 \leftrightarrows 3$ and $2 \leftrightarrows 4$ of the columns of $U$

We take into account that all observable transition probabilities are invariant under the phase transformation

$$
U_{\alpha j} \rightarrow e^{i x_{\alpha}} U_{\alpha j} e^{i y_{j}}
$$

\footnotetext{
${ }^{5}$ These values are compatible with the present upper bound for $B_{e ; e}$, which is given by the results of the Bugey [35] experiment, and with the present allowed range of $A_{\mu ; e}$, which is given by the results of the LSND 15] and Bugey experiments $\left(B_{e ; e} \lesssim 4 \times 10^{-2}\right.$ and $2 \times 10^{-3} \lesssim A_{\mu ; e} \lesssim 4 \times 10^{-2}$ for $\Delta m_{\mathrm{SBL}}^{2}$ in the LSND range (2.6)). Notice that the allowed range of $A_{\mu ; e}$ will be checked in the near future by KARMEN [53] and other experiments [54]. Furthermore, information on $A_{e ; \tau}$ could be obtained in the future in experiments using a $\nu_{e}$ beam from a muon collider (see Refs. [55, 56]).
} 
where $x_{\alpha}$ and $y_{j}$ are arbitrary parameters. Therefore, $U_{e k}$ with $k=3,4$ and $U_{\mu i}$ with $i=1,2$ can always be considered as real vectors. Let us write them as

$$
\begin{aligned}
& U_{e k}=r_{e} v_{k-2} \quad(k=3,4), \\
& U_{\mu i}=p_{\mu} w_{i} \quad(i=1,2),
\end{aligned}
$$

with

$$
r_{e}=\sqrt{\sum_{k=3,4}\left|U_{e k}\right|^{2}} \text { and } p_{\mu}=\sqrt{\sum_{i=1,2}\left|U_{\mu i}\right|^{2}} .
$$

The unit vectors $v$ and $w$ can be written in the form

$$
v=(\cos \theta, \sin \theta) \text { and } \quad w=(\cos \gamma, \sin \gamma)
$$

Let us introduce also the orthogonal unit vectors

$$
v^{\perp}=(-\sin \theta, \cos \theta) \text { and } w^{\perp}=(-\sin \gamma, \cos \gamma) .
$$

Then the vectors $U_{\alpha i}$ with $\alpha \neq e$ and $i=3,4$ can be expanded over the orthonormal basis $\left\{v, v^{\perp}\right\}$ and the vectors $U_{\alpha i}$ with $\alpha \neq \mu$ and $i=1,2$ can be expanded over the orthonormal basis $\left\{w, w^{\perp}\right\}$. As a result, we have the parameterization

$$
U=\left(\begin{array}{ll}
p_{e} w+q_{e} w^{\perp} & r_{e} v \\
p_{\mu} w & r_{\mu} v+s_{\mu} v^{\perp} \\
p_{\tau} w+q_{\tau} w^{\perp} & r_{\tau} v+s_{\tau} v^{\perp} \\
p_{s} w+q_{s} w^{\perp} & r_{s} v+s_{s} v^{\perp}
\end{array}\right)
$$

for the mixing matrix. Using the invariance under the transformation (5.1), we can choose the parameters $q_{\tau}$ and $s_{s}$ as real.

From Eqs.(1.6) and (5.4) it is obvious that the parameters $r_{e}$ and $p_{\mu}$ are connected, respectively, with the parameters $c_{e}$ and $c_{\mu}$ by the relations

$$
r_{e}=\sqrt{1-c_{e}}, \quad p_{\mu}=\sqrt{c_{\mu}} .
$$

Furthermore, from the unitarity of $U$ it follows that the parameters $r_{\mu}$ and $p_{e}$ are related by

$$
r_{\mu}=-p_{e}^{*} \sqrt{\frac{c_{\mu}}{1-c_{e}}} .
$$

Similarly, we can think of $q_{\tau}$ and $s_{s}$ being fixed by the unit length of the lines of $U$ and five of the remaining complex parameters by the orthogonality of different lines. Counting the number of remaining real parameters in $U$ we obtain 10 versus 9 physical real parameters in a $4 \times 4$ unitary mixing matrix. A careful inspection of the residual phase freedom reveals that one of the complex parameters $p_{e}, q_{e}, r_{\mu}, s_{\mu}, p_{\tau}, r_{\tau}, s_{\tau}, p_{s}, q_{s}$ or $r_{s}$ can be chosen real in addition.

The significance of the parameters in the matrix (5.7) shows up by considering the amplitudes of neutrino oscillations in disappearance and appearance SBL experiments, which are directly connected with the parameters $p_{\alpha}$ and $r_{\alpha}$. In fact, we have 


$$
B_{e ; e}=4 r_{e}^{2}\left(1-r_{e}^{2}\right), \quad B_{\mu ; \mu}=4 p_{\mu}^{2}\left(1-p_{\mu}^{2}\right)
$$

and

$$
A_{\mu ; e}=4\left|p_{e}\right|^{2} p_{\mu}^{2}, \quad A_{e ; \tau}=4\left|r_{\tau}\right|^{2} r_{e}^{2}, \quad A_{\mu ; \tau}=4\left|p_{\tau}\right|^{2} p_{\mu}^{2} .
$$

The SBL oscillation amplitudes do not contain the angles $\theta$ and $\gamma$. This is connected with the fact that SBL amplitudes are determined by products of vectors in the 1,2 space or in the 3, 4 space (see Eqs.(2.3) and (2.4)). However, information on the angle $\gamma$ can be obtained from the results of atmospheric and LBL neutrino experiments, whereas the results of solar neutrino experiments give information on the angle $\theta$.

In the four-neutrino scheme $\mathrm{A}$ the parameters $p_{e}, q_{e}$ are small because $c_{e}=\left|p_{e}\right|^{2}+\left|q_{e}\right|^{2}$ is small (see Eq.(2.7)). Let us now investigate if it is possible to obtain bounds on the parameters $\left|p_{s}\right|^{2}$ and $\left|q_{s}\right|^{2}$ from measurable quantities. We will use the unitarity of the mixing matrix and we will work in the approximation $c_{e}=0$. In this case, the parameterization (5.7) of the mixing matrix reduces to

$$
U \simeq\left(\begin{array}{ll}
0 & v \\
p_{\mu} w & s_{\mu} v^{\perp} \\
p_{\tau} w+q_{\tau} w^{\perp} & s_{\tau} v^{\perp} \\
p_{s} w+q_{s} w^{\perp} & s_{s} v^{\perp}
\end{array}\right)
$$

and the amplitude of $\nu_{\mu} \rightarrow \nu_{\tau}$ oscillations is given by (see Eq.(2.3))

$$
A_{\mu ; \tau}=4\left|s_{\mu}\right|^{2}\left|s_{\tau}\right|^{2}
$$

Furthermore, taking into account that

$$
\begin{array}{r}
\left|p_{\tau}\right|^{2}+\left|q_{\tau}\right|^{2}+\left|s_{\tau}\right|^{2}=1 \\
\left|q_{\tau}\right|^{2}+\left|q_{s}\right|^{2}=1
\end{array}
$$

we have

$$
\left|q_{s}\right|^{2}=\left|s_{\tau}\right|^{2}+\left|p_{\tau}\right|^{2} \geq\left|s_{\tau}\right|^{2}
$$

From Eqs.(5.13) and (5.16) and taking into account that $\left|s_{\mu}\right|^{2}=1-c_{\mu} \leq a_{\mu}^{0}$ (see Eq.(2.7)), we obtain

$$
\left|q_{s}\right|^{2} \geq \frac{A_{\mu ; \tau}}{4 a_{\mu}^{0}} .
$$

This lower bound for $\left|q_{s}\right|^{2}=c_{s}-\left|p_{s}\right|^{2}$, obtained in the approximation $a_{e}^{0}=0$, is consistent with the lower bound (4.10) on $c_{s}$. From the comparison of these two lower bounds it is clear that if $c_{s}$ is large, its value approximately coincides with $\left|q_{s}\right|^{2}$, whereas $\left|p_{s}\right|^{2}$ is small if $a_{\mu}^{0}$ is small. Indeed, it is possible to obtain an upper bound for the parameter $\left|p_{s}\right|^{2}$ using the unitarity relation

$$
p_{\mu} p_{s}^{*}+s_{\mu} s_{s}^{*}=0 .
$$


Taking into account that

$$
\begin{aligned}
& \left|s_{\mu}\right|^{2}=1-p_{\mu}^{2}, \\
& \left|s_{s}\right|^{2}=1-\left|p_{s}\right|^{2}-\left|q_{s}\right|^{2},
\end{aligned}
$$

we find from Eq.(5.18)

$$
\frac{\left|p_{s}\right|^{2}}{1-\left|q_{s}\right|^{2}}=1-p_{\mu}^{2} .
$$

Since $1-p_{\mu}^{2}=1-c_{\mu} \leq a_{\mu}^{0}$ (see Eq.(2.7)), we obtain the upper bound

$$
\left|p_{s}\right|^{2} \leq a_{\mu}^{0} .
$$

Therefore, if the experimental upper bound for the amplitude of SBL $\nu_{\mu} \rightarrow \nu_{\mu}$ transitions is small, $\left|p_{s}\right|^{2}$ is small as well.

Note that the observation of SBL inclusive $\nu_{\mu} \rightarrow \nu_{\mu}$ transitions together with the observation of SBL $\nu_{\mu} \rightarrow \nu_{\tau}$ and $\nu_{\mu} \rightarrow \nu_{e}$ (LSND [15]) transitions would allow to get information on the transitions of muon neutrinos into sterile states. In fact, because of the unitarity of the mixing matrix, for the amplitude $A_{\mu ; s}$ of $\nu_{\mu} \rightarrow \nu_{s}$ transitions we have

$$
A_{\mu ; s}=B_{\mu ; \mu}-A_{\mu ; e}-A_{\mu ; \tau} .
$$

Hence, we think that it is important to continue the search for neutrino oscillations in the inclusive $\nu_{\mu} \rightarrow \nu_{\mu}$ channel, especially in the region of small $\Delta m_{\mathrm{SBL}}^{2}$, below $0.3 \mathrm{eV}^{2}$, which has not been explored so far. Let us consider the possibility that the right side of Eq.(5.23) will be measured to be different from zero, i.e., that $\nu_{\mu} \rightarrow \nu_{s}$ transitions occur in SBL experiments. In the scheme under consideration the amplitude of these transitions is given by

$$
A_{\mu ; s}=4 p_{\mu}^{2}\left|p_{s}\right|^{2} .
$$

Taking into account that $p_{\mu}^{2}=c_{\mu}$, Eqs.(4.13), (5.23) and (5.24) imply that

$$
\left|p_{s}\right|^{2}=\frac{B_{\mu ; \mu}-A_{\mu ; e}-A_{\mu ; \tau}}{2\left(1+\sqrt{1-B_{\mu ; \mu}}\right)} .
$$

Finally, if it will be found that not only $\nu_{e} \rightarrow \nu_{e}$ SBL transitions but also $\nu_{\mu} \rightarrow \nu_{\mu}$ SBL transitions are strongly suppressed, it will mean that the only oscillation channel involving sterile neutrinos which may be not suppressed is the channel $\nu_{\tau} \rightarrow \nu_{s}$. Therefore, in this case it will be possible to obtain information on transitions of active neutrinos into sterile states only if $\nu_{\tau}$ beams (see Ref. [57) will be available. In this case $\left|p_{s}\right|^{2} \simeq 0$ and

$$
B_{\tau ; \tau}=A_{\tau ; s}=4\left|q_{s}\right|^{2}\left(1-\left|q_{s}\right|^{2}\right) .
$$

Let us now consider the possibility that not only $p_{e}, q_{e}$ but also $p_{s}, q_{s}$ are small, as follows from the smallness of $c_{s}=\left|p_{s}\right|^{2}+\left|q_{s}\right|^{2}$ implied by BBN if $N_{\nu} \lesssim 3.9$, as shown in Section [II]. In this case it is possible to expand all the quantities $p_{\alpha}, q_{\alpha}, r_{\alpha}$ and $s_{\alpha}$ in powers of the small 
parameters $p_{e}, q_{e}, p_{s}, q_{s}$, up to order $c_{e}, c_{s}, \sqrt{c_{e} c_{s}}$. This means that in this approximation it is possible to express all elements of $U$ in terms of the parameters $p_{e}, q_{e}, p_{s}, q_{s}, \theta$ and $\gamma$. Note that one of the phases of the small complex parameters is unphysical and can be transformed away reaching thus the number of 9 physically independent real parameters in $U$. Performing this program we obtain

$$
\begin{gathered}
\left|p_{\mu}\right|^{2} \simeq 1-\left|p_{e}\right|^{2}-\left|p_{s}\right|^{2}, \quad r_{\mu} \simeq-p_{e}^{*}, \quad s_{\mu} \simeq-p_{s}^{*}, \quad s_{\tau} \simeq-q_{s}^{*}, \\
p_{\tau} \simeq-p_{e} q_{e}^{*}-p_{s} q_{s}^{*}, \quad\left|q_{\tau}\right|^{2} \simeq 1-\left|q_{e}\right|^{2}-\left|q_{s}\right|^{2}, \quad r_{\tau} \simeq-q_{e}^{*}, \quad\left|s_{s}\right|^{2} \simeq 1-c_{s} .
\end{gathered}
$$

In this approximation, Eqs. (5.10) and (5.11) become

$$
B_{e ; e} \simeq 4\left(\left|p_{e}\right|^{2}+\left|q_{e}\right|^{2}\right), \quad B_{\mu ; \mu} \simeq 4\left(\left|p_{e}\right|^{2}+\left|p_{s}\right|^{2}\right)
$$

and

$$
A_{\mu ; e} \simeq 4\left|p_{e}\right|^{2}, \quad A_{e ; \tau} \simeq 4\left|q_{e}\right|^{2}, \quad A_{\mu ; \tau} \simeq 4\left|p_{e} q_{e}^{*}+p_{s} q_{s}^{*}\right|^{2}
$$

Therefore we have

$$
B_{e ; e} \simeq A_{\mu ; e}+A_{e ; \tau}
$$

The absolute value of $p_{s}$ is given by

$$
\left|p_{s}\right|^{2} \simeq \frac{1}{4}\left(B_{\mu ; \mu}-A_{\mu ; e}\right) .
$$

In the absence of $\nu_{\tau}$ disappearance experiments (see Eq.(5.26)), the determination of the parameter $\left|q_{s}\right|$ needs further information from LBL experiments. This is not an artifact of the expansion in Eq.(5.27). The reason is that $B_{\mu ; \mu}$ and $A_{\mu ; \tau}$ are small if $\left|p_{s}\right|$ is small and this is true even if $\left|q_{s}\right|$ is large, i.e. $c_{s}$ is large. This explains the limitations in our search for tests of the BBN constraint on $c_{s}$ derived in Section III. On the other hand, in the present approximation, for the determination of the values of $\left|p_{e}\right|,\left|q_{e}\right|$ and $\left|p_{s}\right|$ in principle the results of SBL experiments alone are sufficient.

The free parameter $\left|p_{e}\right|^{2}$ is fixed by a measurement of the SBL $\nu_{\mu} \leftrightarrows \nu_{e}$ oscillation amplitude. With the result of the LSND experiment and the negative result of all other SBL neutrino oscillation experiments $\left|p_{e}\right|^{2}$ is approximately bounded by $0.5 \times 10^{-3} \lesssim\left|p_{e}\right|^{2} \lesssim 10^{-2}$. If the BBN constraint on $c_{s}$ is valid, then $\nu_{\mu} \rightarrow \nu_{\tau}$ transitions are significantly suppressed in SBL neutrino oscillation experiments with an oscillation amplitude of order $10^{-3}$ or smaller. Furthermore, Eq.(5.30) shows that the oscillation amplitude $A_{e ; \tau}$ is at most close to 0.1.

In the discussion in Section $[1]$ we have seen that the BBN bound $N_{\nu} \lesssim 3.9$ implies that $c_{s} \ll 10^{-2}$. In this case we have

\footnotetext{
${ }^{6}$ Actually, this is always possible because of unitarity, but in general the relations are very complicated.
} 


$$
B_{\mu ; \mu} \simeq A_{\mu ; e} \quad \text { and } \quad A_{\mu ; \tau} \simeq \frac{1}{4} A_{\mu ; e} A_{e ; \tau}
$$

Inspecting Eq.(5.27) we note that in scheme A the quantity $1-c_{\mu}$ is now of the same order of magnitude as $c_{e}$. As mentioned in the previous Section, this is particularly interesting for $\Delta m_{\mathrm{SBL}}^{2} \lesssim 0.3 \mathrm{eV}^{2}$ where the bound on $B_{\mu ; \mu}$ from the $\nu_{\mu}$ disappearance experiments is not very stringent and, consequently, in this $\Delta m_{\mathrm{SBL}}^{2}$ region $B_{\mu ; \mu}$ should be much smaller than the present upper experimental upper bound. In addition, also $1-c_{\tau}$ is of the same order of magnitude, showing that at zeroth-order in the expansion over the small quantities $c_{e}$ and $c_{s}$, the mixing matrix is given by

$$
U \simeq\left(\begin{array}{ll}
0 & v \\
w & 0 \\
w^{\perp} & 0 \\
0 & v^{\perp}
\end{array}\right)
$$

Therefore, we have

$$
\begin{array}{ll}
\nu_{e} \simeq \cos \theta \nu_{3}+\sin \theta \nu_{4}, & \nu_{s} \simeq-\sin \theta \nu_{3}+\cos \theta \nu_{4}, \\
\nu_{\mu} \simeq \cos \gamma \nu_{1}+\sin \gamma \nu_{2}, & \nu_{\tau} \simeq-\sin \gamma \nu_{1}+\cos \gamma \nu_{2} .
\end{array}
$$

Hence, the $\nu_{e}, \nu_{s}-\nu_{3}, \nu_{4}$ and $\nu_{\mu}, \nu_{\tau}-\nu_{1}, \nu_{2}$ sectors are decoupled and the oscillations of solar and atmospheric neutrinos are independent. Furthermore, since only the small mixing angle MSW solutions of the solar neutrino problem seems to be allowed by the data in the case of $\nu_{e} \rightarrow \nu_{s}$ transitions (see Refs. [31, 32, 16]), at zeroth-order in the expansion over the small quantities $c_{e}, c_{s}$ and $\theta$ we have

$$
\nu_{e} \simeq \nu_{3}, \quad \nu_{s} \simeq \nu_{4}
$$

Similar conclusions are valid in scheme B with $c_{\alpha}$ replaced by $1-c_{\alpha}$ and $1,2 \leftrightarrows 3,4$.

Let us now discuss LBL neutrino oscillations. Keeping only the dominant terms we get

$$
P_{\substack{(-) \\ \nu_{\mu} \rightarrow \nu_{\tau}}}^{(\mathrm{LBL})} \simeq 1-P_{\substack{(-) \\ \nu_{\mu} \rightarrow \nu_{\mu}}}^{(\mathrm{LBL})} \simeq \sin ^{2}(2 \gamma) \sin ^{2}(\phi / 2)
$$

where $\phi \simeq \Delta m_{21}^{2} L / 2 p$ in scheme $\mathrm{A}$ and $\Delta m_{43}^{2} L / 2 p$ in scheme B. This shows that there must be significant LBL $\nu_{\mu} \rightarrow \nu_{\tau}$ oscillations because of the sizeable $\nu_{\mu}$ disappearance known from the atmospheric neutrino anomaly, which implies that $\sin ^{2}(2 \gamma)$ is large. For $\nu_{\mu} \rightarrow \nu_{e}$ and $\nu_{e} \rightarrow \nu_{\tau}$ transitions matter effects have to be taken into account. The upper bounds on these transition probabilities derived in Ref. [24] are of order $10^{-2}$. If $c_{s}$ is small, as follows from the BBN constraint, the "matter-stable" bounds on $P_{\substack{(-) \\ \nu_{\mu} \rightarrow \nu_{e}}}^{(\mathrm{LBL})}$ and $P_{\substack{(-) \\ \nu_{e} \rightarrow \underset{\nu}{\nu_{\tau}}}}^{(\mathrm{LBL})}$ derived in Ref. [24 are improved for small values of $\Delta m_{\mathrm{SBL}}^{2}$ because of the inequalities

$$
c_{\mu}, c_{\tau} \geq 1-a_{e}^{0}-c_{s}
$$

leading to

$$
P_{\substack{(-) \\ \nu_{\mu} \rightarrow \stackrel{(-)}{\nu_{e}}}}^{(\mathrm{LBL})}, P_{\substack{(-) \\ \nu_{e} \rightarrow{ }^{(-)}}}^{(\mathrm{LBL})} \leq 2 a_{e}^{0}\left(1-a_{e}^{0}\right)+c_{s}\left(1-2 a_{e}^{0}\right)
$$


Hence, the BBN constraint on $c_{s}$ imply that

$$
P_{\substack{(-) \\ \nu_{\mu} \rightarrow \stackrel{(-)}{\nu_{e}}}}^{(\mathrm{LBL})}, P_{\left(-\nu_{e} \rightarrow \nu_{\tau}\right.}^{(\mathrm{LBL})} \lesssim 2 a_{e}^{0}\left(1-a_{e}^{0}\right)
$$

Obviously, the observation of a violation of these inequalities in LBL neutrino oscillation experiments would imply that the BBN constraint on $c_{s}$ is not valid.

\section{CONCLUSIONS}

In this paper we have focused our discussion on the two four-neutrino schemes $\mathrm{A}$ and $\mathrm{B}$ (see Eq.(1.5) ) which are compatible with the results of all neutrino oscillation experiments 22,23]. As shown in Ref. [23], the bound $N_{\nu} \lesssim 3.9$ for the effective number $N_{\nu}$ of light

neutrinos before the onset of BBN implies a stringent upper bound on $c_{s} \equiv \sum_{k=1,2}\left|U_{s k}\right|^{2}$ in scheme $\mathrm{A}$ and on $1-c_{s}$ in scheme B. Our limits are more stringent than those of Ref. [23] because we used the complete collision rates presented in Ref. [42]. Thus, in scheme A we have obtained $c_{s} \lesssim 5 \times 10^{-3}$. We have demonstrated that in scheme $\mathrm{B}$ the limit derives from resonant transitions due to $\Delta m_{\mathrm{SBL}}^{2}$, leading to an upper bound for $1-c_{s}$ that is more stringent than the one for $c_{s}$ in scheme A: $1-c_{s} \lesssim 5 \times 10^{-5}$.

The validity of these limits from $\mathrm{BBN}$ depends to a certain extent on the results of the measurements of the primordial deuterium abundance which are controversial at the moment [27]. We have therefore emphasized the importance of terrestrial experiments to get information on the value of $c_{s}$ in order to check the BBN constraints. In Section $\amalg \nabla$ we have worked out lower bounds on $c_{s}$ in scheme $\mathrm{A}\left(1-c_{s}\right.$ in scheme $\left.\mathrm{B}\right)$ involving the following observables in SBL experiments:

i. the $\nu_{\mu} \rightarrow \nu_{\tau}$ oscillation amplitude $A_{\mu ; \tau}$;

ii. the oscillation amplitude $B_{\mu ; \mu}$ in $\nu_{\mu}$ disappearance experiments for $\Delta m_{\mathrm{SBL}}^{2} \lesssim 0.3 \mathrm{eV}^{2}$;

iii. the oscillation amplitudes $B_{e ; e}$ in reactor disappearance experiments and $A_{\mu ; e}$ and $A_{e ; \tau}$ in accelerator experiments.

To proceed further, in Section $\square$ we have introduced a parameterization of the $4 \times 4$ neutrino mixing matrix $U$ (5.7) which is particularly suited for the schemes $\mathrm{A}$ and $\mathrm{B}$. In this parameterization the quantity $c_{s}$ is given by $c_{s}=\left|p_{s}\right|^{2}+\left|q_{s}\right|^{2}$ in scheme $\mathrm{A}\left(1-c_{s}=\left|p_{s}\right|^{2}+\left|q_{s}\right|^{2}\right.$ in scheme $\mathrm{B}$ ), where $p_{s}$ and $q_{s}$ are in general complex parameters in $U$. The parameterization (5.7) has lead us to the following observations:

(a) The parameter $\left|p_{s}\right|$ can be determined in SBL $\nu_{\mu} \rightarrow \nu_{e, \mu, \tau}$ oscillation experiments.

(b) For the determination of $\left|q_{s}\right|$ in SBL oscillation experiments it is necessary to employ a $\nu_{\tau}$ neutrino beam and perform a disappearance experiment.

(c) For $N_{\nu} \lesssim 3.9$ the SBL oscillation amplitude $A_{\mu ; \tau}$ is at most of order $10^{-3}$ whereas the LBL oscillation amplitude must be of order one in the same channel. 
As already pointed out in Ref. [23], the BBN constraint $N_{\nu} \lesssim 3.9$ leads to a 4-neutrino mixing picture in which, apart from small corrections of the order of the small quantities $c_{e}$ and $c_{s}$, the mixing matrix $U$ is composed by the two decoupled sectors $\nu_{e}, \nu_{s}-\nu_{3}, \nu_{4}$ and $\nu_{\mu}, \nu_{\tau}-\nu_{1}, \nu_{2}$ in scheme $\mathrm{A}\left(\nu_{e}, \nu_{s}-\nu_{1}, \nu_{2}\right.$ and $\nu_{\mu}, \nu_{\tau}-\nu_{3}, \nu_{4}$ in scheme B). Consequently, the oscillations of solar and atmospheric neutrinos are independent. The solar neutrino problem is solved with $\nu_{e} \rightarrow \nu_{s}$ oscillations governed by the mixing angle $\theta$ and the atmospheric neutrino anomaly is explained with $\nu_{\mu} \rightarrow \nu_{\tau}$ oscillations governed by the mixing angle $\gamma$ (see Eq.(5.7) for the definition of these angles). Concerning the solar neutrino deficit, it was shown that a vacuum oscillation solution involving sterile neutrinos is rather disfavored by the data (see Refs. [31, 32]). Therefore, the specific prediction of a small mixing angle MSW solution of the solar neutrino problem with only $\nu_{e} \rightarrow \nu_{s}$ transitions serves as an indirect check of the schemes A and B under consideration and of the BBN bound. This prediction implies a characteristic strong distortion of the ${ }^{8} \mathrm{~B} \nu_{e}$ spectrum and a day-night asymmetry in the neutral current event rate at SNO [58]. Experimentally, this prediction can be checked independently from solar model calculations (see Ref. [59]).

If indeed the BBN constraints on the 4-neutrino mixing matrix $U$ are confirmed then the biggest gap in our knowledge of $U$, the sterile neutrino mixing, is considerably narrowed. In this paper we have shown that it is possible to test the ensuing mixing matrix to some extent in terrestrial experiments. The most striking feature in this context is the suppression of all SBL oscillation amplitudes. 


\section{REFERENCES}

[1] S.M. Bilenky and B. Pontecorvo, Phys. Rep. 41, 225 (1978).

[2] S.M. Bilenky and S.T. Petcov, Rev. Mod. Phys. 59, 671 (1987).

[3] R.N. Mohapatra and P.B. Pal, Massive Neutrinos in Physics and Astrophysics, World Scientific Lecture Notes in Physics, Vol. 41 (Singapore, 1991).

[4] C.W. Kim and A. Pevsner, Neutrinos in Physics and Astrophysics, Contemporary Concepts in Physics, Vol.8 (Harwood Academic Press, Chur, Switzerland, 1993).

[5] B.T. Cleveland et al., Nucl. Phys. B (Proc. Suppl.) 38, 47 (1995).

[6] K.S. Hirata et al., Phys. Rev. D 44, 2241 (1991).

[7] GALlEX Coll., W. Hampel et al., Phys. Lett. B 388, 384 (1996).

[8] J.N. Abdurashitov et al., Phys. Rev. Lett. 77, 4708 (1996).

[9] K. Inoue, Talk presented at the $5^{\text {th }}$ International Workshop on Topics in Astroparticle and Underground Physics, Gran Sasso, Italy, September 1997 (http://www-sk.icrr.utokyo.ac.jp/doc/sk/pub/pub_sk.html); R. Svoboda, Talk presented at the Conference on Solar Neutrinos: News About SNUs, 2-6 December 1997, Santa Barbara, California (http://www.itp.ucsb.edu/online/snu/).

[10] M. Nakahata, Talk presented at the APCTP Workshop: Pacific Particle Physics Phenomenology, Seoul, Korea, October 31 - November 2, 1997 (http://www-sk.icrr.utokyo.ac.jp/doc/sk/pub/pub_sk.html).

[11] Y. Fukuda et al., Phys. Lett. B 335, 237 (1994).

[12] R. Becker-Szendy et al., Nucl. Phys. B (Proc. Suppl.) 38, 331 (1995).

[13] W.W.M. Allison et al., Phys. Lett. B 391, 491 (1997).

[14] Super-Kamiokande Coll., Y. Fukuda et al., ICRR-Report-411-98-7 (hep-ex/9803006).

[15] C. Athanassopoulos et al., Phys. Rev. Lett. 77, 3082 (1996).

[16] N. Hata and P. Langacker, Phys. Rev. D 56, 6107 (1997).

[17] G.L. Fogli, E. Lisi and D. Montanino, preprint hep-ph/9709473.

[18] M.C. Gonzalez-Garcia et al., preprint hep-ph/9801368.

[19] S.P. Mikheyev and A.Yu. Smirnov, Yad. Fiz. 42, 1441 (1985) [Sov. J. Nucl. Phys. 42, 913 (1985)]; Il Nuovo Cimento C 9, 17 (1986); L. Wolfenstein, Phys. Rev. D 17, 2369 (1978); ibid. 20, 2634 (1979).

[20] J.T. Peltoniemi and J.W.F. Valle, Nucl. Phys. B 406, 409 (1993); D.O. Caldwell and R.N. Mohapatra, Phys. Rev. D 48, 3259 (1993); Z. Berezhiani and R.N. Mohapatra, Phys. Rev. D 52, 6607 (1995); J.R. Primack et al., Phys. Rev. Lett. 74, 2160 (1995); E. Ma and P. Roy, Phys. Rev. D 52, R4780 (1995); R. Foot and R.R. Volkas, Phys. Rev. D 52, 6595 (1995); E.J. Chun et al., Phys. Lett. B 357, 608 (1995); J.J. Gomez-Cadenas and M.C. Gonzalez-Garcia, Z. Phys. C 71, 443 (1996); S. Goswami, Phys. Rev. D 55, 2931 (1997); A.Yu. Smirnov and M. Tanimoto, Phys. Rev. D 55, 1665 (1997); E. Ma, Mod. Phys. Lett. A 11, 1893 (1996).

[21] S.M. Bilenky, C. Giunti, C.W. Kim and S.T. Petcov, Phys. Rev. D 54, 4432 (1996).

[22] S.M. Bilenky, C. Giunti and W. Grimus, Proc. of Neutrino96, Helsinki, June 1996, edited by K. Enqvist et al., p.174 (World Scientific, Singapore, 1997); Eur. Phys. J. C 1, 247 (1998).

[23] N. Okada and O. Yasuda, Int. J. Mod. Phys. A 12, 3669 (1997).

[24] S.M. Bilenky, C. Giunti and W. Grimus, Phys. Rev. D 57 (1998) 1920. 
[25] S.M. Bilenky, C. Giunti and W. Grimus, preprint hep-ph/9712537, to be published in Phys. Rev. D.

[26] V. Barger, T. Weiler and K. Whisnant, preprint hep-ph/9712495; S.C. Gibbons, R.N. Mohapatra, S. Nandi and A. Raychaudhuri, preprint hep-ph/9803299.

[27] C.J. Copi, D.N. Schramm and M.S. Turner, Phys. Rev. Lett. 55, 3389 (1997); N. Hata et al., Phys. Rev. D 55, 540 (1997); C.J. Copi, D.N. Schramm and M.S. Turner, Phys. Rev. D 55, 3389 (1997); D.N. Schramm and M.S. Turner, preprint FERMILAB-Pub97/186-A (astro-ph/9706069); Keith A. Olive, talk given at TAUP97, Gran Sasso Lab., September 1997, preprint astro-ph/9712160.

[28] E.W. Kolb and M.S. Turner, The Early Universe (Addison-Wesley 1990).

[29] R.F. Carswell et al., Mon. Not. R. Astron. Soc. 268, L1 (1994) and 278, 506 (1996); E.J. Wampler et al., Astron. Astrophys. 316, 33 (1996); A. Songaila et al., Nature 368, 599 (1994); M. Rugers and C.J. Hogan, Astrophys. J. Lett. 459, 1 (1996) and Astron. J. 111, 2135 (1996); J.K. Webb et al., Nature 388, 250 (1997).

[30] S. Burles and D. Tytler, preprint astro-ph/9803071, to appear in the Proceedings of the Second Oak Ridge Symposium on Atomic \& Nuclear Astrophysics, (Oak Ridge, TN, December 2-6, 1997), ed. A. Mezzacappa (Institute of Physics, Bristol).

[31] P.I. Krastev and S.T. Petcov, Phys. Rev. Lett. 72, 1960 (1994); Phys. Rev. D 53, 1665 (1996).

[32] S.T. Petcov, Proc. of Neutrino 96, Helsinki, June 1996, edited by K. Enqvist et al., p. 106 (World Scientific, Singapore, 1997).

[33] M. Apollonio et al., preprint hep-ex/9711002.

[34] G.L. Fogli, E. Lisi and G. Scioscia, Phys. Rev. D 56, 3081 (1997).

[35] B. Achkar et al., Nucl. Phys. B 434, 503 (1995).

[36] F. Dydak et al., Phys. Lett. B 134, 281 (1984).

[37] I.E. Stockdale et al., Phys. Rev. Lett. 52, 1384 (1984).

[38] S.M. Bilenky, A. Bottino, C. Giunti and C.W. Kim, Phys. Rev. D 54, 1881 (1996).

[39] K. Kainulainen, Phys. Lett. B 224, 191 (1990).

[40] R. Barbieri and A. Dolgov, Phys. Lett. B 237, 440 (1990).

[41] K. Enqvist, K. Kainulainen and J. Maalampi, Nucl. Phys. B 349, 754 (1991).

[42] K. Enqvist, K. Kainulainen and M. Thomson, Nucl. Phys. B 373, 498 (1992).

[43] D. Nötzold and G. Raffelt, Nucl. Phys. B 307, 924 (1988).

[44] J.M. Cline, Phys. Rev. Lett. 68, 3137 (1992).

[45] K. Enqvist, K. Kainulainen and J. Maalampi, Phys. Lett B 249, 531 (1990).

[46] X. Shi, D. N. Schramm and B. D. Fields, Phys. Rev. D 48, 2563 (1993).

[47] L. D. Landau, Phys. Z. USSR 1, 426 (1932); C. Zener, Proc. R. Soc. A 137, 696 (1932).

[48] R. Foot, M.J. Thomson and R.R. Volkas, Phys. Rev. D 53, 5349 (1996); X. Shi, Phys. Rev. D 54, 2753 (1996); R. Foot and R.R. Volkas, Phys. Rev. D 55, 5147 (1997).

[49] D. Macina, Nucl. Phys. B (Proc. Suppl.) 48, 183 (1996).

[50] M. Laveder, Nucl. Phys. B (Proc. Suppl.) 48, 188 (1996).

[51] R.A. Sidwell, Proc. of Neutrino96, Helsinki, June 1996, edited by K. Enqvist et al., p.152 (World Scientific, Singapore, 1997).

[52] G. Brooijmans, talk presented at Aspen 29/1/98 (http://choruswww.cern.ch/Reference/).

[53] J. Kleinfeller, Nucl. Phys. B (Proc. Suppl.) 48, 207 (1996). 
[54] L. Ludovici and P. Zucchelli, preprint CERN-PPE/96-181; Booster Neutrino Experiment (BooNE), http://nu1.lampf.lanl.gov/BooNE.

[55] S. Geer, preprint Fermilab-PUB-97/389 (hep-ph/9712290).

[56] R.N. Mohapatra, Talk presented at the Workshop on Physics with the First Muon Collider, Fermilab, 6-9 November 1997, preprint hep-ph/9711444.

[57] F. Vannucci, Proc. of the $5^{\text {th }}$ International Workshop on Neutrino Telescopes, Venezia, March 1993, p.57.

[58] P.I. Krastev, Q.Y. Liu and S.T. Petcov, Phys. Rev. D 54, 7057 (1996).

[59] S.M. Bilenky and C. Giunti, Phys. Lett. B 311, 179 (1993); Phys. Lett. B 320, 323 (1994); Astropart. Phys. 2, 353 (1994); Z. Phys. C 68, 495 (1995). 


\section{FIGURES}

FIG.1. Plot in the $A_{\mu ; \tau}-\Delta m_{\mathrm{SBL}}^{2}$ plane of the curve $4\left(a_{\mu}^{0}\right)^{2}$ (solid line). For $A_{\mu ; \tau}$ to the right of this curve (see Eq.(4.8)) the lower bound (4.9) for $c_{s}$ applies. The dashed curve representing $B_{\mu ; \mu}^{0}$ constitutes an upper bound for $A_{\mu ; \tau}$ due to the conservation of probability. The values of $a_{\mu}^{0}$ and $B_{\mu ; \mu}^{0}$ have been obtained from the 90\% CL exclusion curves of the CDHS [36] and CCFR [37] experiments. The dash-dotted curve, dash-dotdotted and dotted curves represent, respectively, the most recent exclusion curve presented by the CHORUS collaboration [52], the expected final sensitivity of the CHORUS [49] and NOMAD [50] experiments and the expected sensitivity of the COSMOS [51] experiment.

FIG.2. Plot in the $c_{s}-\Delta m_{\mathrm{SBL}}^{2}$ plane of the lower limit $2 a_{\mu}^{0}-a_{e}^{0}$ for $c_{s}$ that follows from the inequality (4.8). The values of $a_{e}^{0}$ and $a_{\mu}^{0}$ have been obtained from the 90\% CL exclusion curves of the Bugey [35], CDHS [36] and CCFR [37] experiments.

FIG.]3. Plot in the $A_{\mu ; \tau}-\Delta m_{\text {SBL }}^{2}$ plane of the upper bound $\left(a_{e}^{0}\right)^{2}$ (solid curve) for $A_{\mu ; \tau}$ (see Eq.(4.11) in the case of a very small $c_{s}$. The values of $a_{e}^{0}$ have been obtained from the $90 \%$

CL exclusion curve of the Bugey [35] experiment. The dash-dotted curve, dash-dot-dotted and dotted curves are the same as in Fig.1. 


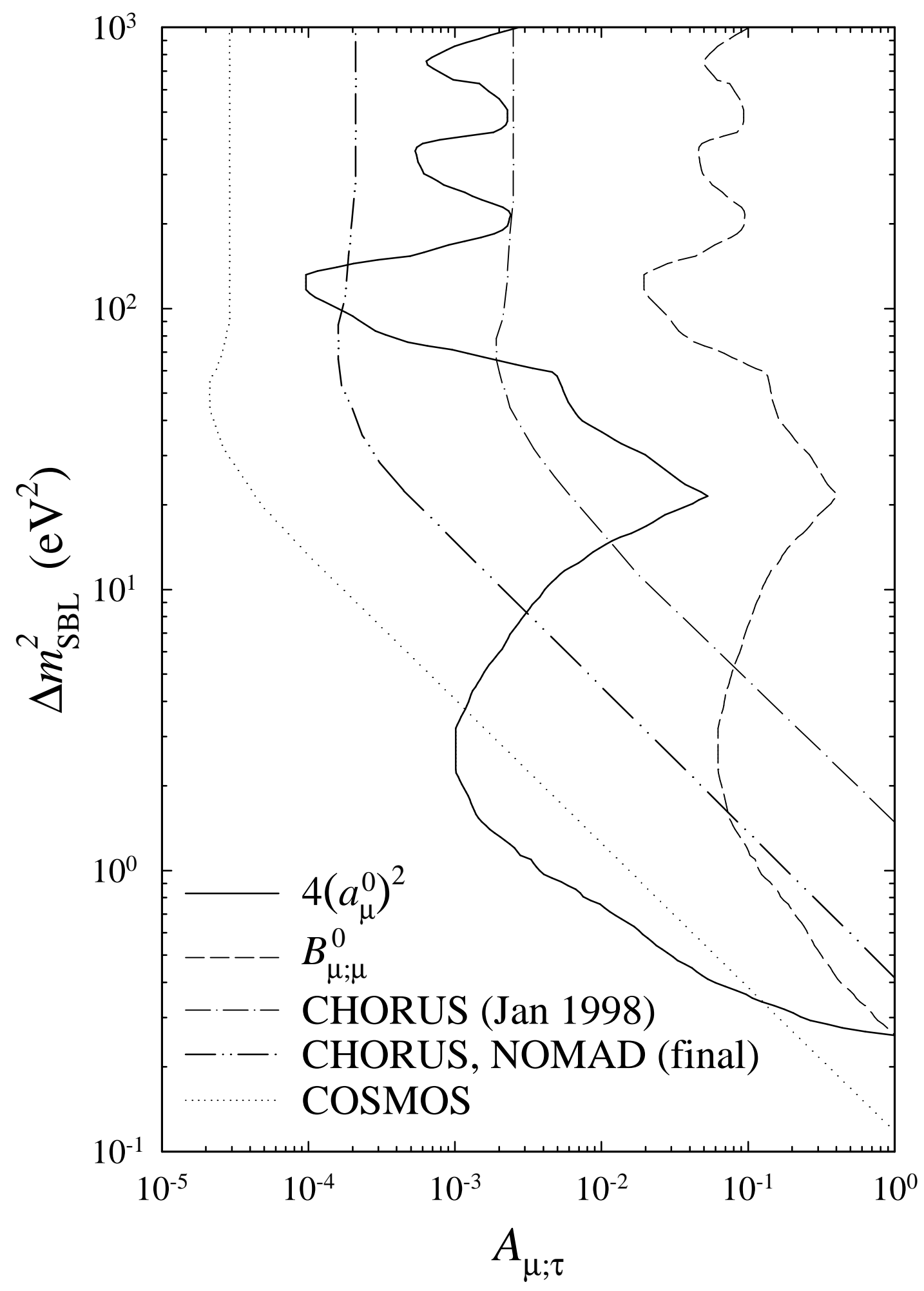

Figure 凹 


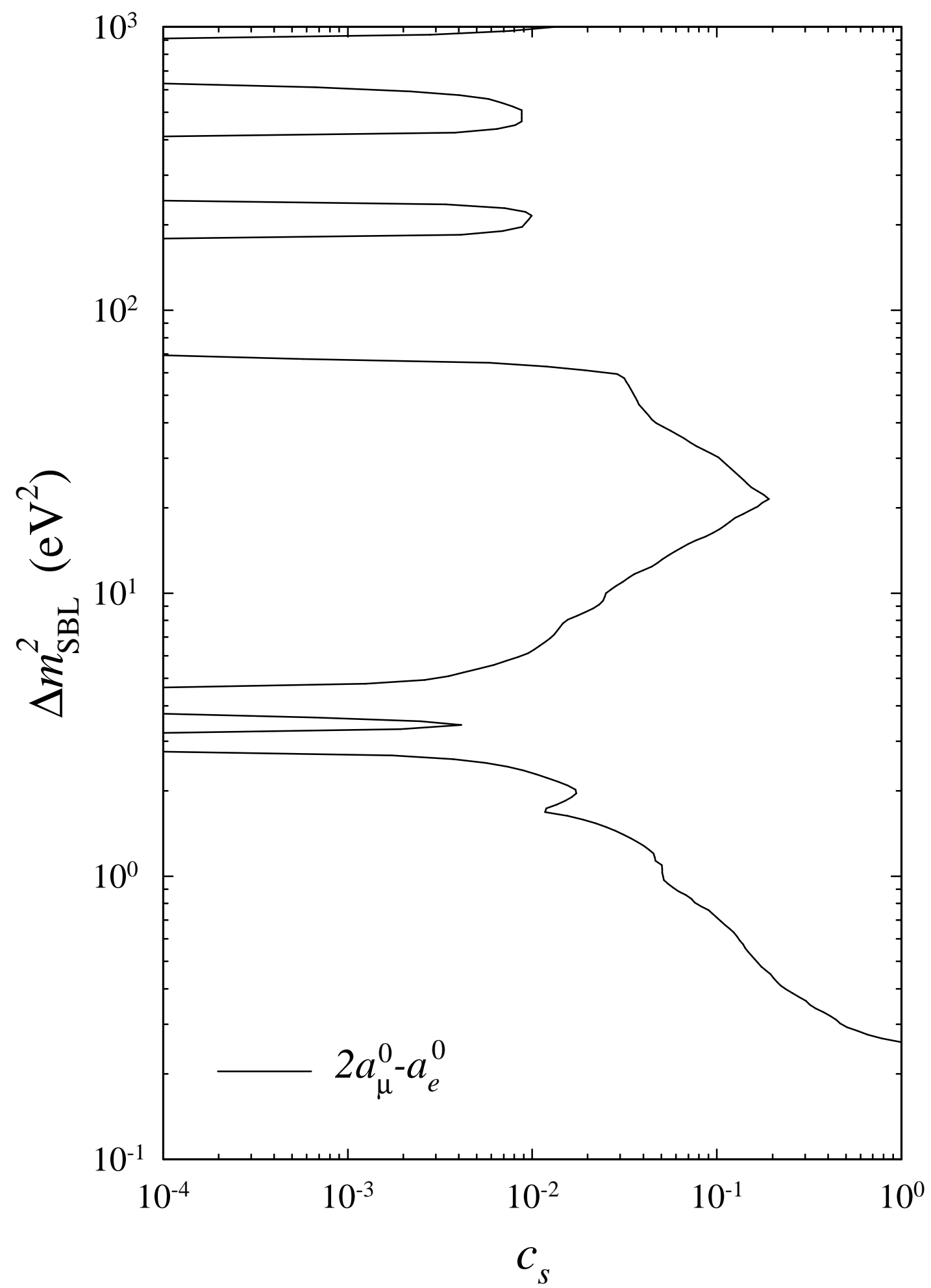

Figure 2 


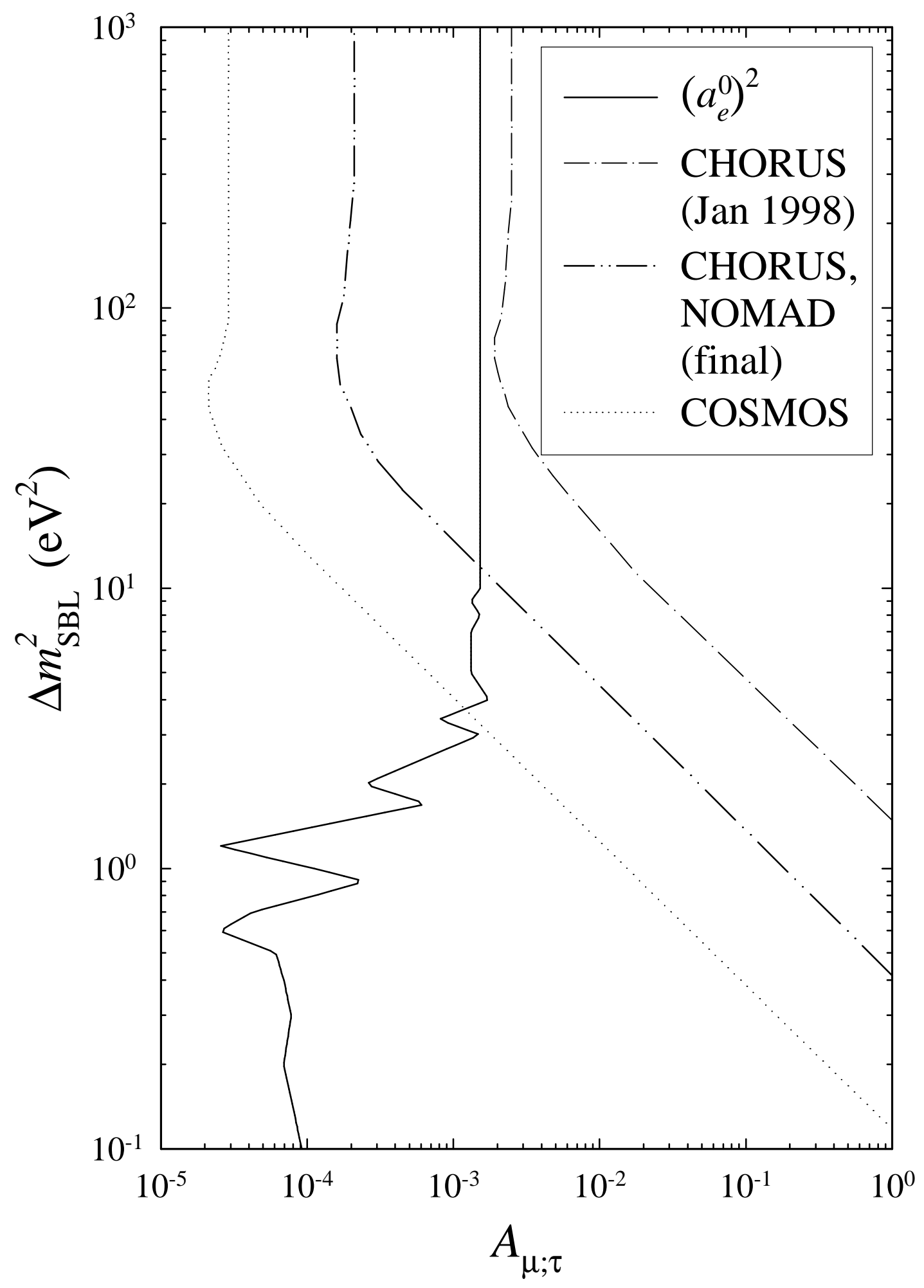

Figure 3 